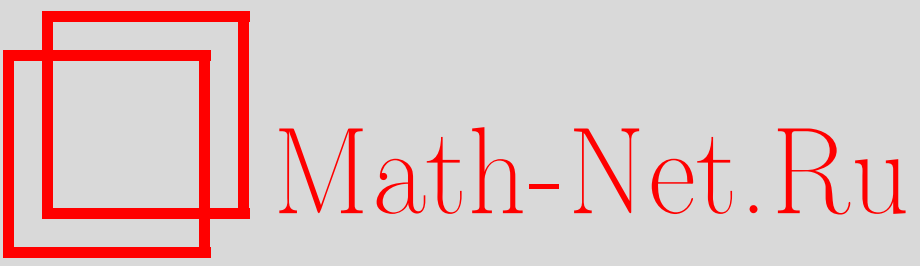

А. С. Криницын, В. В. Прудников, П. В. Прудников, Расчет динамического критического индекса методом суммирования асимптотических рядов, ТМФ, 2006, том 147, номер 1, 137-154

DOI: https://doi.org/10.4213/tmf2029

Использование Общероссийского математического портала Math-Net.Ru подразумевает, что вы прочитали и согласны с пользовательским соглашением

http://www.mathnet.ru/rus/agreement

Параметры загрузки:

IP : 35.173 .219 .149

26 апреля 2023 г., 12:35:01 
ТЕОРЕТИЧЕСКАЯ

И МАТЕМАТИЧЕСКАЯ

ФИЗИКА

Том 147, № 1

апрель, 2006

(C) 2006 г.

А. С. Криницын*, В.В. Прудников*, П. В. Прудников*

\section{РАСЧЕТ ДИНАМИЧЕСКОГО КРИТИЧЕСКОГО ИНДЕКСА МЕТОДОМ СУММИРОВАНИЯ АСИМПТОТИЧЕСКИХ РЯДОВ}

Рассмотрено применение методов суммирования асимптотических рядов Паде-Бореля, Паде-Бореля-Лероя и конформного отображения к расчету динамического критического индекса для однородных и неупорядоченных изингоподобных систем.

Ключевые слова: методы суммирования, асимптотические ряды, критическая динамика, ренормализационная группа, динамический критический индекс.

\section{1. ВВЕДЕНИЕ}

Данная работа посвящена изложению деталей применения методов суммирования асимптотических рядов для расчета критического индекса $z$, определяющего критическое замедление времени релаксации системы $\tau \sim \xi^{z} \sim\left|T-T_{\mathrm{c}}\right|^{-z \nu}$ вбли-

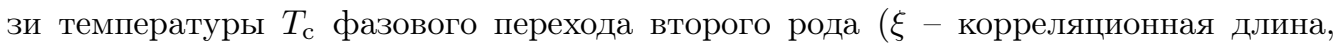
$\nu$ - индекс корреляционной длины). Наиболее сложным и интересным направлением теории критических явлений является исследование динамических процессов в окрестности критической точки. Существенным достижением ренормализационногруппового (РГ) подхода в исследовании статических критических явлений явилось создание концепции классов универсальности критического поведения различных систем, характеризующихся сходными критическими свойствами. Универсальность динамических критических явлений в отличие от статических значительно слабее, что проявляется в существовании большого числа моделей критической динамики с различным динамическим критическим поведением [1] при общих равновесных критических свойствах. Эта трудность частично компенсируется тем, что динамические критические характеристики многих из этих моделей могут быть выражены через характеристики их статического критического поведения. В первую очередь это касается моделей, в которых выполняются законы сохранения параметра порядка или

* Омский государственный университет, Омск, Россия. E-mail: krinitsin@univer.omsk.su; prudnikv@univer.omsk.su; prudnikp@univer.omsk.su 
других долгоживущих возбуждений, в частности плотности энергии [1]. Наличие сохраняющихся величин может приводить к возникновению в стохастических уравнениях движения чисто гидродинамических слагаемых, определяющих, а иногда и подавляющих влияние флуктуационных процессов на динамическое (релаксационное) поведение. В этом случае критические индексы, описывающие динамическое критическое поведение, могут быть вычислены на основе статических индексов с точностью определения последних [1].

Однако существуют динамические модели, описание критического поведения которых не является тривиальной задачей. В первую очередь к ним относятся модели с чисто диссипативными уравнениями движения. Для исследования критического поведения таких моделей приходится развивать специальные методы и проводить расчеты динамических величин независимо от статических. Среди этих моделей наибольший интерес представляет динамическая модель Гинзбурга-Ландау (модель $A$ в классификационной схеме Гальперина и Хоенберга [1]), впервые предложенная Ландау и Халатниковым для описания аномального затухания звука в гелии вблизи $\lambda$-точки. Впоследствии в рамках этой модели стали описывать динамическое критическое поведение других реальных систем, таких как изингоподобные магнетики.

Критическая динамика модели $A$ исследовалась РГ-методами, основанными на $\epsilon$-разложении, в статьях [2]. В работах [3], [4] одного из авторов этой статьи впервые для ее описания был развит теоретико-полевой подход непосредственно для трехмерных и двумерных однородных систем последовательно в 3-петлевом и 4петлевом приближениях, а затем и для структурно-неупорядоченных трехмерных изингоподобных систем в 3-петлевом приближении [5]. Однако достигнутая в цитированных работах точность определения динамических критических характеристик все же значительно уступает точности описания статического критического поведения для данных систем, осуществленного в 6-петлевом приближении теории [6]-[9]. В первую очередь это обусловлено быстрым ростом объема вычислений уже в самых низких порядках теории возмущений, особенно при описании критической динамики структурно-неупорядоченных систем. В связи с этим при описании критической динамики модели $A$ в однородных и неупорядоченных системах возрастают требования к точности тех методов, которые применяются для суммирования асимптотических рядов теории при получении значения динамического критического индекса $z$.

В данной работе впервые представлены результаты применения к рядам теории методов суммирования Паде-Бореля-Лероя (ПБЛ) и конформного отображения $(\mathrm{KO})$ для вычисления значений индекса $z$. Проведено сопоставление полученных результатов с экспериментальными данными и результатами компьютерного моделирования критической динамики методами Монте-Карло.

\section{2. МОДЕЛЬ}

Для описания аномальных свойств термодинамических характеристик систем при фазовых переходах второго рода широко применяется метод теоретико-полевой РГ, позволяющий рассчитать значения критических индексов, характеризующих асимптотическое поведение термодинамических и корреляционных функций вблизи 
критической температуры.

Рассматриваемая модель критического поведения однородной ферромагнитной системы представляет собой классическую спиновую систему, термодинамически эквивалентную $O(n)$-симметричной модели Гинзбурга-Ландау с эффективным гамильтонианом

$$
\mathcal{H}=\frac{1}{2} \int d^{d} x\left(|\nabla \varphi|^{2}+r_{0} \varphi^{2}+\frac{g_{0}}{12} \varphi^{4}\right),
$$

где $d$ - размерность системы, $\varphi(\vec{x}, t)-n$-компонентный параметр порядка (намагниченность $), r_{0} \sim T-T_{\mathrm{c} 0} \quad\left(T_{\mathrm{c} 0}-\right.$ критическая температура в приближении среднего поля) и $g_{0}>0$ - вершина взаимодействия флуктуаций намагниченности. Динамическое поведение магнетика в релаксационном режиме вблизи критической точки в рамках модели $A$ описывается кинетическим уравнением для параметра порядка типа уравнения Ланжевена:

$$
\frac{\partial \varphi}{\partial t}=-\lambda_{0} \frac{\delta \mathcal{H}}{\delta \varphi}+\zeta+\lambda_{0} h
$$

$\lambda_{0}$ - кинетический козффициент, $\zeta(\vec{x}, t)$ - гауссова случайная сила, $h(\vec{x}, t)$ - внешнее магнитное поле. Известно, что его решение в виде корреляционных функций и функций отклика можно получить, используя производящий функционал следующего вида:

$$
\Omega=\int D[\varphi] D[\tilde{\varphi}] \exp \left(-\mathcal{H}_{\mathrm{eff}}[\varphi, \tilde{\varphi}]+\int(\varphi h+\tilde{\varphi} \tilde{h}) d^{d} x d t\right)
$$

где введены вспомогательное поле $\tilde{\varphi}$ с источником поля $\tilde{h}$ и функционал действия

$$
\mathcal{H}_{\mathrm{eff}}=\int d^{d} x d t\left(\lambda_{0}^{-1} \tilde{\varphi}^{2}+i \tilde{\varphi}\left(\lambda_{0}^{-1} \frac{\partial \varphi}{\partial t}+\frac{\delta \mathcal{H}}{\delta \varphi}\right)\right) .
$$

При этом функция отклика параметра порядка на поле $h$ определяется как

$$
G(x, t)=\left.\frac{\delta\langle\varphi(x, t)\rangle}{\delta h(x, t)}\right|_{h=0}=\frac{1}{\Omega} \frac{\delta^{2} \Omega}{\delta h(x, t) \delta \tilde{h}(0,0)}=\langle\varphi(x, t) \tilde{\varphi}(0,0)\rangle .
$$

Вместо функции отклика удобнее рассматривать ее вершинную часть $\Gamma^{(1,1)}(k, \omega)$.

Учет дальнодействующих и долгоживущих флуктуаций параметра порядка, во многом определяющих аномалии равновесных и неравновесных характеристик систем при $T \rightarrow T_{\text {c }}$, осуществляется в рамках разработанных РГ-методов [1], [10]. Используемые в теории перенормированные динамические вершинные функции $\Gamma_{\mathrm{R}}^{(N, \widetilde{N})}(k, \omega) \quad(N+\widetilde{N})$-го порядка, однозначно определяющие все наблюдаемые характеристики системы, задаются дифференциальным РГ-уравнением

$$
\left[\mu \frac{\partial}{\partial \mu}+\beta \frac{\partial}{\partial g}-r \gamma_{r} \frac{\partial}{\partial r}-\lambda \gamma_{\lambda} \frac{\partial}{\partial \lambda}-\frac{(N+\tilde{N})}{2} \gamma_{\varphi}\right] \Gamma_{\mathrm{R}}^{(N, \tilde{N})}=0
$$

с перенормированными зарядом $g$, приведенной температурой $r$ и кинетическим коэффициентом $\lambda$. Асимптотическое поведение термодинамических и корреляционных 
функций, имеющее при $T \rightarrow T_{\mathrm{c}}$ степенной характер, определяется нулем $g^{*}$ функции $\beta(g): \quad \beta\left(g^{*}\right)=0$, и значениями функций $\gamma_{r}\left(g^{*}\right), \gamma_{\lambda}\left(g^{*}\right), \gamma_{\phi}\left(g^{*}\right)$, задающими статические и динамический критические индексы: $\nu=\left(2+\gamma_{r}\left(g^{*}\right)\right)^{-1}, \eta=\gamma_{\varphi}\left(g^{*}\right)$, $z=2+\gamma_{\lambda}\left(g^{*}\right)$ (как пример, $\gamma=\nu(2-\eta)$, магнитная восприимчивость $\chi \sim\left|T-T_{\mathrm{c}}\right|^{-\gamma}$ ).

Функции $\beta(g), \gamma_{r}(g), \gamma_{\lambda}(g), \gamma_{\phi}(g)$, входящие в дифференциальное уравнение РГ, могут быть вычислены в виде рядов по $g$. Для размерности пространства $d$, близкой к четырем, координата неподвижной точки $g^{*}$ функции $\beta(g)$ принимает малые значения. В этом случае применимы методы теории возмущений по константе связи $g \sim 4-d$ и можно подсчитать критические индексы. Для реальных систем с $d=3,2$ ряды по $g$ являются асимптотическими, и для их суммирования нужно применять специальные методы, не основанные на представлениях теории возмущений [6], [7].

При описании критического поведения структурно-неупорядоченных систем с замороженными в узлах решетки немагнитными атомами примеси или вакансиями, играющими роль точечных дефектов структуры, в эффективный гамильтониан модели Гинзбурга-Ландау (1) вводят дополнительное слагаемое

$$
\Delta \mathcal{H}[\varphi, V]=\frac{1}{2} \int d^{d} x V(x) \varphi^{2},
$$

где $V(x)$ - потенциал случайного поля дефектов, приводящий к флуктуациям локальной критической температуры $r_{0} \sim T-T_{\mathrm{c0}}$. Распределение дефектов структуры в объеме системы полагается гауссовым (учет отклонения от гауссового распределения приводит лишь к несущественным в критической области поправкам) с функцией распределения

$$
P[V]=A_{V} \exp \left[-\left(16 v_{0}\right)^{-1} \int d^{d} x V^{2}(x)\right],
$$

где $A_{V}$ - нормировочный фактор, $v_{0}$ - положительный параметр, пропорциональный концентрации дефектов и квадрату величины их потенциала. Получаемые в дальнейшем с помощью производящего функционала корреляционные функции и функции отклика для структурно-неупорядоченных систем должны быть усреднены по случайному потенциалу дефектов $V(x)$. Это усреднение наиболее эффективно может быть выполнено с помощью метода реплик, широко используемого в подобных целях при исследованиях слабо неупорядоченных систем (см., например, обзор [11]). Суть метода заключается в формальном применении при получении свободной энергии неупорядоченной системы через процедуру усреднения по распределению дефектов следующего математического преобразования:

$$
-\frac{F}{T}=\langle\langle\ln Z\rangle\rangle=\lim _{m \rightarrow 0} \frac{1}{m} \ln \left\langle\left\langle Z^{m}\right\rangle\right\rangle=\lim _{m \rightarrow 0} \frac{1}{m} \ln \operatorname{Sp}_{\left\{\varphi_{i}\right\}} \exp \left(-\widehat{H}_{\text {repl }} \frac{\left[\left\{\varphi_{i}\right\}\right]}{T}\right),
$$

где двойные угловые скобки обозначают усреднение по распределению вероятностей различных конфигураций дефектов $P[V], Z$ - статистическая сумма исходной неупорядоченной системы и введены $m$ реплик (“образов") исходного поля $\varphi-\left\{\varphi_{i}\right\}$ 
с репличными индексами $i=1, \ldots, m$. При этом репличный гамильтониан определяется как

$$
\exp \left(-\frac{\widehat{H}_{\mathrm{repl}}^{(m)}\left[\left\{\varphi_{i}\right\}\right]}{T}\right)=\left\langle\left\langle\prod_{i=1}^{m} \exp \left(-\frac{\widehat{H}\left[\left\{\varphi_{i}\right\}\right]}{T}\right)\right\rangle\right\rangle
$$

и в отличие от исходного является трансляционно-инвариантным. При применении данной процедуры усреднения к динамическому производящему функционалу (3) вместо функционала действия однородной модели (4) может быть получен репличный функционал действия

$$
\begin{gathered}
\widehat{H}_{\mathrm{repl}}^{(m)}=\sum_{i} \int d^{d} x d t\left[-\lambda_{0}^{-1} \tilde{\varphi}_{i} \tilde{\varphi}_{i}+i \tilde{\varphi}_{i}\left(\lambda_{0}^{-1} \frac{\partial \varphi_{i}}{\partial t}-\nabla^{2} \varphi_{i}+r_{0} \varphi_{i}\right)+\frac{i}{3 !} g_{0} \tilde{\varphi}_{i} \varphi_{i} \varphi_{i} \varphi_{i}\right]+ \\
+4 v_{0} \sum_{i, j} \int d^{d} x d t d t^{\prime} \tilde{\varphi}_{i}(x, t) \varphi_{i}(x, t) \tilde{\varphi}_{j}\left(x, t^{\prime}\right) \varphi_{j}\left(x, t^{\prime}\right) .
\end{gathered}
$$

В соответствии с методом реплик свойства исходной неупорядоченной системы получаются в пределе $m \rightarrow 0$. Данный предел приводит к уничтожению связанных диаграмм, содержащих петли, образованные примесной вершиной $v_{0}$.

Таким образом, учет структурной неупорядоченности систем при описании критического поведения приводит к введению дополнительной вершины взаимодействия $v_{0}$ в эффективном гамильтониане, определяющей эффекты взаимодействия флуктуаций $n m$-компонентного параметра порядка через поле дефектов. Последующая процедура перенормировки динамических вершинных функций задается дифференциальным РГ-уравнением

$$
\left[\mu \frac{\partial}{\partial \mu}+\beta_{g} \frac{\partial}{\partial g}+\beta_{v} \frac{\partial}{\partial v}-r \gamma_{r} \frac{\partial}{\partial r}-\lambda \gamma_{\lambda} \frac{\partial}{\partial \lambda}-\frac{(N+\tilde{N})}{2} \gamma_{\varphi}\right] \Gamma_{\mathrm{R}}^{(N, \tilde{N})}=0
$$

с перенормированными зарядами $g, v$. В этом случае функции $\beta_{g}$ и $\beta_{v}$ зависят от $g$ и $v$ как от параметров модели. Неподвижные точки $\left(g^{*}, v^{*}\right)$ РГ-преобразований определяются нулями функций $\beta_{g}$ и $\beta_{v}: \beta_{g}\left(g^{*}, v^{*}\right)=0, \beta_{v}\left(g^{*}, v^{*}\right)=0$, а критические индексы - значениями функций $\gamma_{r}\left(g^{*}, v^{*}\right), \gamma_{\varphi}\left(g^{*}, v^{*}\right)$ и $\gamma_{\lambda}\left(g^{*}, v^{*}\right)$ в соответствующих устойчивых неподвижных точках РГ-преобразования. Как и для однородных систем, функции $\beta_{g}, \beta_{v}, \gamma_{r}, \gamma_{\lambda}(g)$ и $\gamma_{\phi}(g)$ могут быть вычислены в виде рядов по $g$ и $v$ лишь для размерности системы $d$, близкой к четырем, а для реальных систем с $d=3$ ряды по $g$ и $v$ являются лишь асимптотически сходящимися.

\section{3. МЕТОДЫ СУММИРОВАНИЯ АСИМПТОТИЧЕСКИХ РЯДОВ}

Для обсуждения динамического критического поведения однородных систем и вычисления индекса $z$ нам потребуются только функции $\beta(g), \gamma_{\lambda}(g)$. Явный вид первой из них в 6-петлевом приближении для трехмерных систем и в 4-петлевом приближении для двумерных систем был получен в работах [6], [7]. Нами был проведен расчет динамических скейлинговых функций $\gamma_{\lambda}$ в 4-петлевом приближении для двумерной и трехмерной моделей Изинга [4]. В результате функции $\beta(g)$ и $\gamma_{\lambda}(g)$ 
представляются в виде следующих рядов по заряду $g$ : для двумерной модели

$$
\begin{gathered}
\frac{\beta(g)}{g}=-1+g-0.716174 g^{2}+0.930767 g^{3}-1.582388 g^{4}, \\
\gamma_{\lambda}(g)=0.027053 g^{2}-0.004184 g^{3}+0.022130 g^{4},
\end{gathered}
$$

для трехмерной

$$
\begin{gathered}
\frac{\beta(g)}{g}=-1+g-0.422497 g^{2}+0.351070 g^{3}-0.376527 g^{4}+ \\
+0.495548 g^{5}-0.749689 g^{6} \\
\gamma_{\lambda}(g)=0.008399 g^{2}-0.000045 g^{3}+0.020423 g^{4} .
\end{gathered}
$$

На основе метода, предложенного Липатовым [12], в работе [13] было показано, что, хотя общий член ряда для функции $\beta(g)$ растет факториально:

$$
\beta(g)=\sum_{n=0}^{\infty} c_{n} g^{n}, \quad c_{n} \approx c(-a)^{n} n^{b} n !\left[1+O\left(\frac{1}{n}\right)\right],
$$

тем не менее ряд удовлетворяет условию асимптотической сходимости

$$
\left|\beta(g)-\sum_{n=0}^{N} c_{n} g^{n}\right| \leqslant C \sigma^{N+1}[(N+1) !]^{\sigma}|g|^{N+1}
$$

в клиновидной области $|\arg g| \leqslant(\pi / 2) \sigma$ на комплексной плоскости; $C, \sigma-$ некоторые константы. Сшивка асимптотики (15) со значениями первых коэффициентов дает информацию обо всех членах ряда и позволяет приближенно восстановить функцию $\beta(g)$, однако это требует специальной процедуры суммирования асимптотически сходящихся рядов. Для суммирования подобных рядов разработаны специальные методы [6]-[9], [14], из которых наиболее эффективными являются методы Паде-Бореля (ПБ), ПБЛ и КО. В работах [15] техника Липатова оценки высоких порядков теории обобщается на критическую динамику и доказывается, что асимптотика Липатова остается справедливой и для динамической РГ-функции $\gamma_{\lambda}$. Поэтому указанные методы суммирования, которые успешно зарекомендовали себя при анализе статических РГ-функций, могут быть соответственно применены и для анализа динамических РГ-функций.

Для неупорядоченной трехмерной модели Изинга полученные представления функций $\beta_{g}, \beta_{v}$ и $\gamma_{\lambda}$ в виде рядов по зарядам $g$ и $v$ имеют вид

$$
\begin{gathered}
\frac{\beta_{g}}{g}=-1+g+\frac{3}{2} v-\frac{308}{729} g^{2}-\frac{104}{81} g v-\frac{185}{216} v^{2}+\sum_{i+j \geqslant 3} b_{i, j}^{(g)} g^{i} v^{j}, \\
\frac{\beta_{v}}{v}=-1+\frac{2}{3} g+v-\frac{92}{729} g^{2}-\frac{50}{81} g v-\frac{95}{216} v^{2}+\sum_{i+j \geqslant 3} b_{i, j}^{(v)} g^{i} v^{j}, \\
\gamma_{\lambda}=\sum_{i, j=0}^{3} \gamma_{i, j} g^{i} v^{j}=-\frac{1}{4} v+0.008400 g^{2}+0.030862 g v+0.053240 v^{2}- \\
\quad-0.012642 g^{3}-0.041167 g^{2} v-0.152964 g v^{2}-0.049995 v^{3},
\end{gathered}
$$


где коэффициенты $b_{i, j}^{(g)}, \quad b_{i, j}^{(v)}$ и $\gamma_{i, j}$ вычислены в работах [9] и [5] в 6-петлевом и 3-петлевом приближениях, соответственно.

Предварительно рассмотрим применение методов суммирования ПБ, ПБЛ и КО на примере точно решаемой задачи об определении энергии основного состояния линейного ангармонического осциллятора с гамильтонианом

$$
\mathcal{H}=p^{2}+x^{2}+g x^{4}
$$

Это позволит выделить особенности применения каждого из методов суммирования, сопоставить точность применяемых аппроксимаций в зависимости от числа учитываемых членов рядов. В соответствии с работой [16] энергия основного состояния $E_{0}(g)$ осциллятора может быть представлена в виде ряда теории возмущений по константе ангармонизма $g$, первые члены которого имеют вид

$E_{0}(g)=\sum_{n=0}^{\infty} c_{n} g^{n}=1+\frac{3}{4} g-\frac{21}{16} g^{2}+\frac{333}{64} g^{3}-\frac{30885}{1024} g^{4}+\frac{916731}{4096} g^{5}-\frac{65518401}{32768} g^{6}+\cdots$,

а точные значения $E_{0}$ в зависимости от $g$ приведены в статье [17]. В работе [16] показано, что общий член ряда (19) растет факториально аналогично выражению (15).

Идея метода ПБ состоит в том, что ряд типа (19) представляется интегралом

$$
f(g)=\int_{0}^{\infty} e^{-t} B(g t) d t, \quad B(g)=\sum_{n=0}^{\infty} B_{n} g^{n}, \quad B_{n}=\frac{c_{n}}{n !} .
$$

В этом выражении $B(g)$ носит название борелевского образа. Борелевский образ в соответствии с асимптотикой (15) сходится в круге радиуса $1 / a$. K $B(g)$ затем применяется аппроксимация Паде, состоящая в использовании для $B(g)$ рациональной функции вида

$$
\left[\frac{L}{M}\right]=\frac{\sum_{i=0}^{L} a_{i} g^{i}}{\sum_{j=0}^{M} b_{j} g^{j}}, \quad M \geqslant 1,
$$

разложение которой в ряд Тейлора (в окрестности точки $g=0$ ) совпадает с разложением борелевского образа до тех пор, пока это возможно. Функция вида (21) имеет $L+1$ коэффициентов в числителе и $M+1$ коэффициентов в знаменателе, так что весь набор коэффициентов определяется с точностью до общего множителя, и для определенности полагают $b_{0}=1$. В результате получается всего $L+M+1$ свободных параметров. Это означает, что в общем случае коэффициенты разложения функции $[L / M]$ в ряд Тейлора должны совпадать с соответствующими коэффициентами ряда (19). Если ряд имеет конечное число членов ряда $N$, на аппроксиманты $[L / M]$ накладывается условие $L+M \leqslant N$.

Обобщением метода ПБ является метод ПБЛ, для которого формулы (20) записываются в расширенной форме:

$$
f(g)=\int_{0}^{\infty} t^{b} e^{-t} B(g t) d t, \quad B(g)=\sum_{n=0}^{\infty} B_{n} g^{n}, \quad B_{n}=\frac{c_{n}}{\Gamma(n+b+1)},
$$




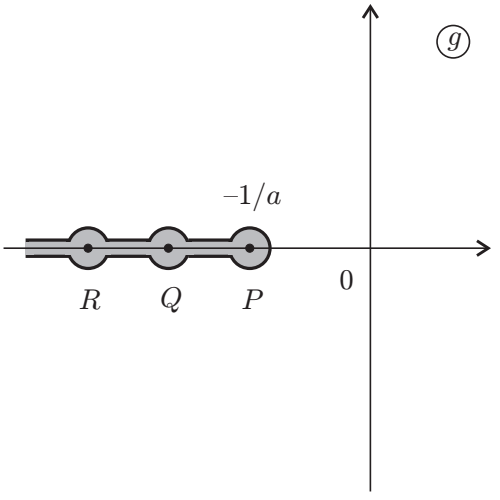

a

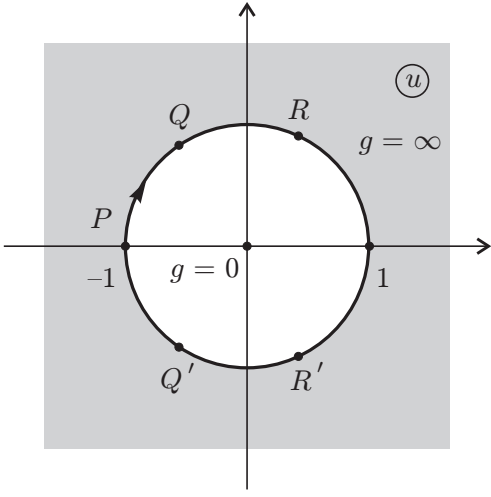

б

Рис. 1. Область аналитичности борелевского образа на комплексной плоскости с разрезом $(-\infty,-1 / a)$ вдоль вещественной оси (а) и ее конформное отображение на единичный круг (б).

где $b$ - произвольный параметр. В простейшем случае реализации метода, например, рассматриваются значения $b=0$ и $b=1$, а затем анализируются изменения в аппроксимациях ряда за счет изменения параметра $b$, которые позволяют сделать вывод о погрешностях аппроксимаций методами ПБ и ПБЛ. В нашем случае применения метода ПБЛ к рядам, определяющим характеристики критического поведения однородных и неупорядоченных систем, выбран в качестве тестового ряд (19) для точно решаемой задачи об энергии ангармонического осциллятора с асимптотической сходимостью ряда, аналогичной сходимости рядов (13), (14) теории критических явлений. Поэтому при применении метода ПБЛ к рядам (13), (14) теории критических явлений планируется использовать те значения параметра $b$, которые приведут к наилучшим аппроксимациям ряда (19).

Рассмотрим основные принципы применения метода КО к борелевскому образу (при $b=0)$. В соответствии с асимптотикой (15) борелевский образ аналитичен в комплексной плоскости $g$ с разрезом от $-1 / a$ до $-\infty$ (рис. 1a). Так как интегрирование в формулах (22) осуществляется по всей вещественной оси, то возникает необходимость в аналитическом продолжении $B(g)$ за пределы круга сходимости $|g|<1 / a$ на произвольные комплексные $g$, реализуемом конформным преобразованием $g=w(u)$ с

$$
w(u)=\frac{4}{a} \frac{u}{(1-u)^{2}}, \quad u=\frac{(1+a g)^{1 / 2}-1}{(1+a g)^{1 / 2}+1},
$$

отображающим плоскость с разрезом на единичный круг $|u|<1$ (рис. 1б). Таким образом, представление $B(g)$ в виде ряда по $u$ дает сходящийся ряд при любых $g$ :

$$
B(g)=\left.\sum_{n=0}^{\infty} B_{n} g^{n}\right|_{g=w(u)} \quad \Longrightarrow \quad B(u)=\sum_{n=0}^{\infty} U_{n} u^{n} .
$$

Действительно, все возможные особые точки $P, Q, R, \ldots$ функции $B(g)$ лежат вдоль 


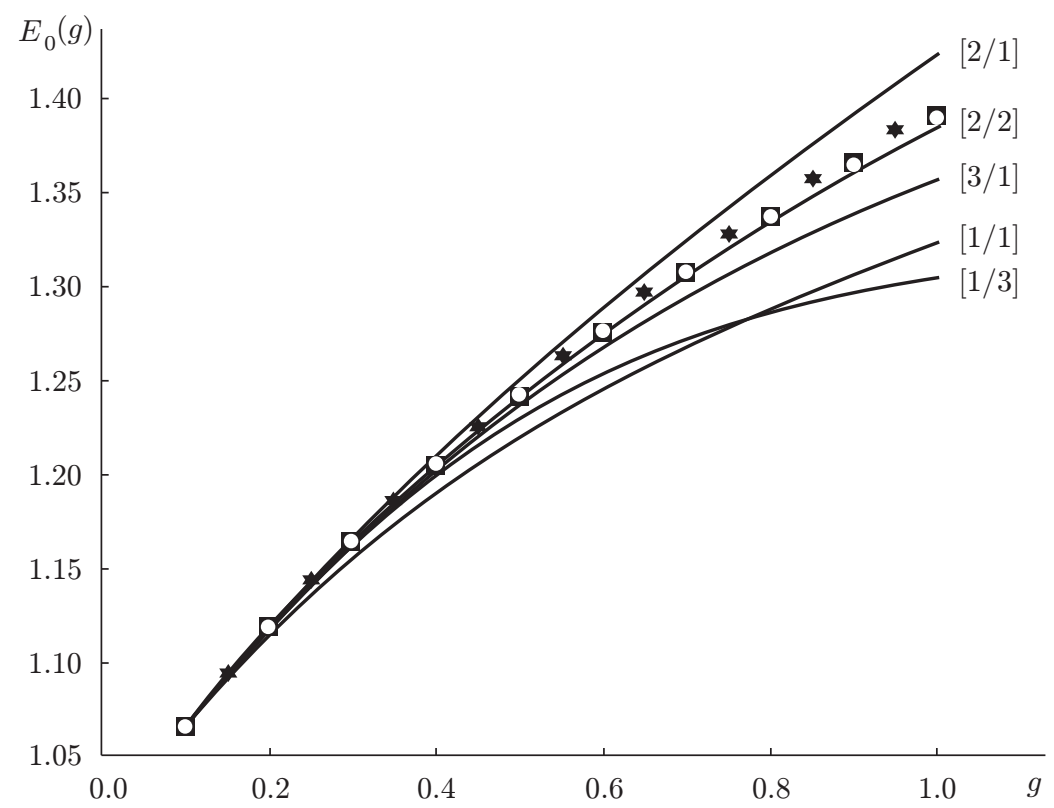

Рис. 2. Сопоставление точных значений энергии основного состояния ангармонического осциллятора $E_{0}$ (черные квадраты) для различных значений константы ангармонизма $g$ с результатами применения методов суммирования ПБ с различными $[L / M]$ (сплошные линии), ПБЛ с отобранным значением параметра $b=2.221426$ для аппроксиманта [1/1] (белые кружки) и КПБ со значением постоянной $a=3$ (звездочки).

разреза, а их образы $P, Q, Q^{\prime}, R, R^{\prime}, \ldots$ - на границе круга $|u|=1$, так что второй из рядов $(24)$ сходится при всех $|u|<1$. Связь коэффициентов $U_{n}$ и $B_{n}$ выражается следующим образом:

$$
U_{0}=B_{0}, \quad U_{n}=\sum_{m=1}^{n} B_{m}\left(\frac{4}{a}\right)^{m} C_{n+m-1}^{n-m}, \quad n \geqslant 1 .
$$

Эти формулы решают поставленную задачу: борелевский образ $B(u(g))$ сходится для любых особых точек $g=\infty, g=-1 / a$ и $g=g_{0}$ с $g_{0} \in(-\infty,-1 / a)$, а коэффициенты $U_{n}$ для него связаны линейным преобразованием (25) с исходными коэффициентами $c_{n}$ и параметром $b$ (см. формулы $\left.(22)\right)$. Поскольку для любых $g$ переменная $|u|<1$, то к ряду по переменной $u$ можно эффективно применять аппроксимацию Паде. Назовем такую процедуру методом конформного Паде-Бореля (КПБ).

На рис. 2 представлено сравнение применяемых к ряду для энергии основного состояния ангармонического осциллятора аппроксимаций с точными значениями $E_{0}$ при использовании метода ПБ с различными типами аппроксимант Паде $[L / M]$, а также методов ПБЛ и КПБ (при $a=3$ [16]) с аппроксимантой [1/1]. Из рис. 2 видно, что погрешность аппроксимации методом ПБ сильно растет с увеличением 
значений $g$, хотя ситуация улучшается с ростом числа учитываемых членов ряда $N$ и с использованием диагональных и близких к диагональному виду аппроксимант. Методы же ПБЛ при $b=2.221426$ (в выражении (26)) и КПБ даже для аппроксиманты $[1 / 1]$ дают результаты, близкие к точным значениям $E_{0}$ в рассматриваемом интервале изменений переменной $g$. С ними оказываются сравнимыми по точности лишь результаты использования “лучшей” диагонального вида аппроксиманты $[2 / 2]$ в методе ПБ.

При реализации метода ПБЛ интегральное преобразование Бореля удобнее осуществлять в следующем виде:

$$
f(g)=\int_{0}^{\infty} d t e^{-t} B\left(g t^{b}\right), \quad B(g)=\sum_{n=0}^{\infty} B_{n} g^{n}, \quad B_{n}=\frac{c_{n}}{\Gamma(b n+1)},
$$

где параметр $b$ для всех $[L / M]$ подбирается из требования, чтобы среднее квадратичное отклонение аппроксимаций от точных значений $E_{0}$ на всем интервале изменения $g$ было минимальным. Использование выражения (26) позволяет добиться значительно лучшего соответствия точному значению $E_{0}$, чем использование $(22)$. При этом данное преобразование в отличие от преобразования (22) позволяет при указанном выше вариационном способе подбора значений $b$ избежать появления особенностей в подынтегральном выражении. В табл. 1 представлены значения суммы квадратов отклонений $S^{2}$ для вычисленных значений энергии осциллятора $E_{0}$ при применении различных аппроксимаций от точных значений $E_{0}$ для методов ПБ, ПБЛ и КПБ. В табл. 1 не приведены результаты применения аппроксимант типа $[0 / N]$, так как было выявлено, что для четных $N$ их использование приводит к очень большим отклонениям суммы ряда от точного решения и отсутствию в погрешности $S^{2}$ минимума по $b$ (наблюдается лишь небольшое уменьшение $S^{2}$ с ростом $b$ ), а для нечетных $N$ возникают неустранимые для любых значений $b$ особенности в борелевском образе, соответствующем данной аппроксиманте. Для аппроксимант типа $[1 / N-1]$ было найдено по два значения параметра $b$, характеризуемых близкими значениями погрешности $S^{2}$. Сопоставление приведенных значений $S^{2}$ наглядно показывает более высокую точность применения метода ПБЛ по сравнению с методом ПБ. Лишь начиная с аппроксимант пятого порядка, близких к диагональному виду, метод ПБ позволяет получать значения, сравнимые по точности с результатами применения метода ПБЛ. Это делает метод ПБЛ более предпочтительным для анализа коротких рядов, полученных при описании критической динамики однородных (13), (14) и неупорядоченных систем (17). Отметим также, что метод КПБ является вторым по точности среди рассмотренных методов и не уступает по точности методу ПБЛ, начиная с аппроксимант пятого порядка по $N$ (за исключением случая применения аппроксиманты [4/2]). Метод ПБ, уступая по точности методу КПБ для коротких рядов, позволяет получать сравнимые с ним результаты лишь для диагональной аппроксиманты $[2 / 2]$ и близких к диагональному виду аппроксимант [3/2] и $[4 / 2]$.

Рассмотрим теперь применение описанных выше методов к расчету динамического критического индекса $z$ для однородной модели Изинга, описывающей критическое поведение систем с размерностями $d=2$ и $d=3$. Как отмечалось выше, 
ТАБЛИЦА 1. Значения сумм квадратов отклонений вычисленных значений энергии осциллятора $E_{0}$ при применении методов ПБ, ПБЛ и КПБ от точных значений $E_{0}$ (пробелы отражают наличие полюса в борелевском образе при использовании соответствующей аппроксиманты)

\begin{tabular}{|c|cccccccc|}
\hline$[L / M]$ & {$[1 / 1]$} & {$[1 / 2]$} & {$[2 / 1]$} & {$[1 / 3]$} & {$[2 / 2]$} & {$[3 / 1]$} & {$[1 / 4]$} & {$[2 / 3]$} \\
\hline$S_{\text {ПБ }}^{2}$ & 0.013923 & - & 0.002703 & 0.016687 & 0.000105 & 0.002535 & - & - \\
\hline$b$ & 2.221426 & 1.582184 & 1.466092 & 1.276639 & 1.131538 & 1.194466 & 2.016677 & 1.050884 \\
& & 3.441754 & & 3.879020 & & & 4.083434 & \\
$S_{\text {ПБл }}^{2}$ & 0.000011 & 0.000006 & 0.000003 & 0.000007 & $3 \cdot 10^{-7}$ & 0.000001 & 0.000010 & 0.000044 \\
& & 0.000010 & & 0.000008 & & & 0.000007 & \\
\hline$S_{\text {КПБ }}^{2}$ & 0.000175 & 0.041795 & 0.000836 & 0.000307 & 0.000192 & - & - & 0.000011 \\
\hline \hline$[L / M]$ & {$[3 / 2]$} & {$[4 / 1]$} & {$[0 / 6]$} & {$[1 / 5]$} & {$[2 / 4]$} & {$[3 / 3]$} & {$[4 / 2]$} & {$[5 / 1]$} \\
\hline$S_{\text {ПБ }}^{2}$ & 0.000001 & 0.004142 & 2.609852 & 0.031233 & - & - & 0.000247 & 0.000833 \\
\hline$b$ & 1.005754 & 1.068052 & $\nexists$ & 2.445345 & 0.970348 & 0.959599 & 0.964316 & 1.006032 \\
& & & & 4.194370 & & 2.003840 & & \\
$S_{\text {ПБл }}^{2}$ & $3 \cdot 10^{-7}$ & 0.000004 & & 0.000007 & $2 \cdot 10^{-7}$ & $2 \cdot 10^{-7}$ & $2 \cdot 10^{-7}$ & 0.000008 \\
& & & & 0.000006 & & $2 \cdot 10^{-7}$ & & \\
\hline$S_{\text {КПь }}^{2}$ & - & - & 0.000064 & - & $3 \cdot 10^{-7}$ & $5 \cdot 10^{-7}$ & 0.003932 & 0.000003 \\
\hline
\end{tabular}

индекс $z$ определяется при использовании функций $\beta(g)$ и $\gamma_{\lambda}(g)$. Неподвижная точка $g^{*}$ РГ-преобразований находится из условия $\beta\left(g^{*}\right)=0$. В табл. 2 приведены значения неподвижной точки $g^{*}$ и динамического критического индекса $z$ для двумерной и трехмерной моделей Изинга, полученные методами ПБ, ПБЛ (при значениях параметра $b$ для соответствующих аппроксимант в задаче осциллятора) и КПБ, где для последнего постоянная $a$ в соответствии с работами [7] принималась равной 0.238659217 для $d=2$ и 0.14777422 для $d=3$. В методах ПБ и ПБЛ при суммировании ряда для функции $2+\gamma_{\lambda}$ использовалась наилучшая (суммируемая) для них аппроксиманта четвертого порядка [3/1], а в методе КПБ оказались несуммируемыми все аппроксиманты, отличные от $[N / 0]$, в результате чего был реализован лишь простой метод КО.

Процедура усреднения результатов, получаемых при применении метода ПБ с учетом аппроксимант порядка $N \geqslant 4$, позволила получить следующие значения заряда $g^{*}$ в неподвижной точке и индекса $z$ :

$$
\begin{array}{ll}
g^{*}=1.7987 \pm 0.0201, & z=2.0847 \pm 0.0019, \quad d=2, \\
g^{*}=1.4270 \pm 0.0074, \quad z=2.0171 \pm 0.0002, \quad d=3 ;
\end{array}
$$

при применении метода ПБЛ -

$$
\begin{aligned}
& g^{*}=1.7012 \pm 0.0115, \quad z=2.0757 \pm 0.0010, \quad d=2, \\
& g^{*}=1.4125 \pm 0.0046, \quad z=2.0168 \pm 0.0001, \quad d=3 ;
\end{aligned}
$$


ТАБЛИцА 2. Значения неподвижной точки $g^{*}$ и динамического критического индекса $z$ для двумерной и трехмерной моделей Изинга, полученные методами ПБ, ПБЛ и КПБ

\begin{tabular}{|c|c|c|c|c|c|c|c|c|c|}
\hline & & {$[L / M]$} & {$[1 / 1]$} & {$[1 / 2]$} & {$[2 / 1]$} & {$[1 / 3]$} & {$[3 / 1]$} & {$[1 / 4]$} & {$[2 / 3]$} \\
\hline \multirow{6}{*}{$d=2$} & \multirow[t]{2}{*}{ ПБ } & $g^{*}$ & 2.1971 & - & 1.8210 & 1.8271 & 1.7702 & & \\
\hline & & $z$ & 2.1262 & & 2.0868 & 2.0873 & 2.0820 & & \\
\hline & \multirow[t]{2}{*}{ ПБЛ } & $g^{*}$ & 1.6978 & 1.7098 & - & 1.7174 & 1.6849 & & \\
\hline & & $z$ & 2.0753 & 2.0764 & & 2.0771 & 2.0742 & & \\
\hline & \multirow{2}{*}{ КПБ } & $g^{*}$ & 2.1448 & - & 1.9137 & - & 1.8193 & & \\
\hline & & $z$ & 2.1270 & & 2.1018 & & 2.0922 & & \\
\hline \multirow{6}{*}{$d=3$} & \multirow[t]{2}{*}{ ПБ } & $g^{*}$ & 1.5508 & - & 1.4314 & 1.4230 & 1.4175 & 1.4427 & 1.4753 \\
\hline & & $z$ & 2.0202 & & 2.0172 & 2.0170 & 2.0169 & 2.0175 & 2.0183 \\
\hline & \multirow[t]{2}{*}{ ПБЛ } & $g^{*}$ & 1.3972 & 1.4019 & 1.4671 & 1.4056 & 1.4049 & 1.4073 & - \\
\hline & & $z$ & 2.0166 & 2.0165 & & 2.0166 & 2.0166 & 2.0166 & \\
\hline & \multirow[t]{2}{*}{ КПБ } & $g^{*}$ & 1.5385 & - & 1.4552 & - & 1.4267 & 1.4253 & - \\
\hline & & $z$ & 2.0452 & & 2.0393 & & 2.0374 & 2.0373 & \\
\hline & & {$[L / M]$} & {$[3 / 2]$} & {$[4 / 1]$} & {$[1 / 5]$} & {$[2 / 4]$} & {$[3 / 3]$} & {$[4 / 2]$} & {$[5 / 1]$} \\
\hline \multirow{6}{*}{$d=3$} & \multirow[t]{2}{*}{ ПБ } & $g^{*}$ & 1.4234 & 1.4292 & - & 1.4263 & - & 1.4200 & 1.3854 \\
\hline & & $z$ & 2.0170 & 2.0172 & & 2.0171 & & 2.0169 & 2.0161 \\
\hline & \multirow[t]{2}{*}{ ПБЛ } & $g^{*}$ & 1.4234 & 1.4355 & 1.4082 & - & 1.4221 & 1.4208 & 1.3848 \\
\hline & & $z$ & 2.0170 & 2.0173 & 2.0167 & & 2.0170 & 2.0170 & 2.0161 \\
\hline & \multirow[t]{2}{*}{ КПБ } & $g^{*}$ & 1.4242 & 1.4245 & - & - & - & - & 1.4147 \\
\hline & & $z$ & 2.0372 & 2.0373 & & & & & 2.0366 \\
\hline
\end{tabular}

при использовании метода КПБ -

$$
\begin{array}{lll}
g^{*}=1.8193, & z=2.0922, & d=2, \\
g^{*}=1.4231 \pm 0.0019, & z=2.0372 \pm 0.0001, & d=3 .
\end{array}
$$

Отметим, что все полученные значения заряда $g^{*}$ в неподвижной точке для $d=3$ хорошо согласуются с результатом работы Ле Гийу и Зин-Жюстина [7]: $g^{*}=1.416 \pm$ 0.005 .

Усреднение приведенных выше значений, полученных разными методами, дает итоговые величины:

$$
\begin{array}{lll}
g^{*}=1.7731 \pm 0.0297, & z=2.0842 \pm 0.0039, & d=2, \\
g^{*}=1.4209 \pm 0.0035, & z=2.0237 \pm 0.0055, & d=3 .
\end{array}
$$

В случае описания критического поведения неупорядоченных систем ряды теории 
для функций $\beta_{g}, \beta_{v}$ и $\gamma_{\lambda}(17)$ будут двухпараметрическими:

$$
F(v, g)=\sum_{i, j} C_{i, j} v^{i} g^{j}, \quad i, j=0,1,2, \ldots
$$

$\mathrm{K}$ таким рядам методы, описанные выше, напрямую применять нельзя. Они нуждаются в модификации на случай нескольких переменных. Один из методов, допускающих такую возможность, носит название $\lambda$-метода. Суть его заключается во введении обобщенного ряда

$$
\widetilde{F}(v, g ; \lambda)=\sum_{n=0}^{\infty} \widetilde{C}_{n}(v, g) \lambda^{n}, \quad \widetilde{C}_{n}(v, g)=\sum_{i, j} C_{i, j} v^{i} g^{j} \delta_{i+j, n}
$$

который при $\lambda=1$ сводится к исходному ряду (27). Такой ряд можно считать зависящим от одной переменной $\lambda$, коэффициенты разложения в котором являются функциями $u$ и $v$. Преобразование Бореля ряда (27) имеет вид

$$
F(v, g)=\int_{0}^{\infty} d t e^{-t} B\left(v t^{b}, g t^{b}\right), \quad B(v, g)=\sum_{i, j} \frac{C_{i, j}}{\Gamma((i+j) b+1)} v^{i} g^{j}
$$

Борелевский образ с помощью $\lambda$-метода сводится к зависимости лишь от одной переменной $\lambda$, к которому затем применяется метод аппроксимант Паде и осуществляется вычисление интеграла.

При применении метода КО к двухпараметрическим рядам используется замена $\delta=v / u$, с помощью которой ряд (27) можно переписать как

$$
\left.F(v, g)\right|_{g=\delta v}=\sum_{n=0}^{\infty} \widetilde{C}_{n}(\delta) v^{n}
$$

Преобразование Бореля при этом принимает вид

$$
F(v, \delta)=\int_{0}^{\infty} d t e^{-t} B\left(v t^{b}, \delta\right), \quad B(v, \delta)=\sum_{n=0}^{\infty} \frac{\widetilde{C}_{n}(\delta)}{\Gamma(n b+1)} v^{n}
$$

Конформное преобразование определяется формулой

$$
u(v)=\frac{\sqrt{1+\alpha(\delta) v}-1}{\sqrt{1+\alpha(\delta) v}+1} .
$$

При использовании метода КО к рядам теории критического поведения неупорядоченных систем задача усложняется тем, что асимптотика рядов в этом случае не известна. В работе [9] для модели Изинга предлагается $\alpha(\delta)$ брать в следующем виде:

$$
\alpha(\delta)=a\left(\frac{9}{8}+\delta\right), \quad \delta<0, \quad \delta>4
$$

где для трехмерной модели Изинга $a=0.14777422$. 
Рассмотрим теперь применение этих двух методов к рядам (17), характеризующим неравновесное критическое поведение неупорядоченной модели Изинга. В табл. 3 приведены результаты применения данных методов суммирования к расчету координат неподвижной точки, задающей критическое поведение неупорядоченных систем, и индекса $z$. В методах ПБ и ПБЛ при суммировании ряда для функции $2+\gamma_{\lambda}$ использовалась наилучшая для них аппроксиманта третьего порядка [2/1], а в методе КПБ - аппроксиманта $[1 / 2]$. Из табл. 3 видно, что все эти методы дают близкие значения для вершин в неподвижных точках и индекса $z$.

ТАБлицА 3. Результаты применения методов ПБ, ПБЛ и КПБ к расчету неподвижных точек и динамического критического индекса $z$ для неупорядоченной модели Изинга

\begin{tabular}{|c|c|c|c|c|c|c|c|c|c|}
\hline$[L / M]$ & $\begin{array}{c}N=2 \\
{[1 / 1]}\end{array}$ & $\begin{array}{c}N=3 \\
{[1 / 2]}\end{array}$ & $\begin{array}{c}N=4 \\
{[2 / 2]}\end{array}$ & {$[3 / 1]$} & $\begin{array}{c}N=5 \\
{[1 / 4]}\end{array}$ & {$[3 / 2]$} & {$[4 / 1]$} & $\begin{array}{c}N=6 \\
{[4 / 2]}\end{array}$ & {$[5 / 1]$} \\
\hline ПБ & & & & & & & & & \\
\hline$v^{*}$ & -0.5868 & -0.6894 & -0.7383 & -0.6991 & -0.6394 & -0.7146 & -0.7201 & -0.7125 & \\
\hline$g^{*}$ & 2.3900 & 2.2402 & 2.2743 & 2.2411 & 2.2497 & 2.2640 & 2.2705 & 2.2583 & \\
\hline$z$ & 2.1509 & 2.1756 & 2.1878 & 2.1780 & 2.1633 & 2.1819 & 2.1833 & 2.1814 & \\
\hline \multicolumn{10}{|l|}{ ПБЛ } \\
\hline$v^{*}$ & -0.6862 & -0.7001 & -0.7000 & -0.7142 & -0.6900 & -0.7142 & -0.7089 & -0.7141 & \\
\hline$g^{*}$ & 2.3895 & 2.2499 & 2.2499 & 2.2459 & 2.2601 & 2.2638 & 2.2646 & 2.2600 & \\
\hline$z$ & 2.1757 & 2.1787 & 2.1787 & 2.1822 & 2.1762 & 2.1823 & 2.1809 & 2.1822 & \\
\hline \multicolumn{10}{|l|}{ КПБ } \\
\hline$v^{*}$ & & -0.6800 & -0.6700 & -0.6808 & -0.7302 & -0.7239 & & -0.7321 & -0.7321 \\
\hline$g^{*}$ & & 2.1654 & 2.1342 & 2.2562 & 2.3079 & 2.2671 & & 2.2763 & 2.2799 \\
\hline$z$ & & 2.1716 & 2.1691 & 2.1710 & 2.1844 & 2.1831 & & 2.1854 & 2.1853 \\
\hline
\end{tabular}

Применим процедуру усреднения к полученным результатам, используя все значения аппроксимант $[L / M]$, кроме [1/1]. Ниже приведены средние значения координат неподвижной точки и индекса $z$, полученные с использованием метода ПБ:

$$
v^{*}=-0.7019 \pm 0.0111, \quad g^{*}=2.2569 \pm 0.0048, \quad z=2.1788 \pm 0.0027
$$

метода ПБЛ:

$$
v^{*}=-0.7059 \pm 0.0033, \quad g^{*}=2.2563 \pm 0.0026, \quad z=2.1802 \pm 0.0008 ;
$$

метода КПБ:

$$
v^{*}=-0.7070 \pm 0.0100, \quad g^{*}=2.2410 \pm 0.0227, \quad z=2.1786 \pm 0.0026 .
$$

Полученные различными методами близкие значения координат неподвижной точки и индекса $z$, практически совпадающие в пределах погрешностей, говорят о 
достоверности полученных результатов. Усреднение данных значений, полученных разными методами, дает итоговые величины:

$$
v^{*}=-0.7049 \pm 0.0013, \quad g^{*}=2.2514 \pm 0.0042, \quad z=2.1792 \pm 0.0004 .
$$

В работе [5] при расчете динамического критического индекса $z$ с применением метода Чисхолма-Бореля было получено значение $z=2.1653$. Это значение индекса неплохо коррелирует с полученными в настощей работе результатами.

\section{4. ЗАКЛЮЧЕНИЕ}

В данной работе впервые осуществлено последовательное применение методов суммирования ПБ, ПБЛ и КО с последующим использованием аппроксимации Паде (метод КПБ) для определения значений динамического критического индекса $z$ для однородных двумерных и трехмерных систем, описываемых моделью Изинга, а также неупорядоченных изингоподобных систем. Анализ был осуществлен на доступных к настоящему времени лишь коротких рядах теории, соответствующих результатам 4-петлевого описания критической динамики однородных систем и 3 петлевого описания неупорядоченных систем [4], [5].

Проведем сопоставление полученных результатов с экспериментальными данными и результатами компьютерного моделирования критической динамики методами Монте-Карло. Критическая динамика однородного изинговского антиферромагнетика $\mathrm{FeF}_{2}$ экспериментально была исследована в работе [18], и было получено значение $z=2.1 \pm 0.1$, которое в рамках достаточно широкого интервала погрешности измерения $z$ не противоречит нашим результатам теоретико-полевого расчета с использованием методов суммирования. Численные исследования методами Монте-Карло критической динамики однородной трехмерной модели Изинга в соответствии с различными литературными данными привели к следующим значениям динамического критического индекса: $z=1.99 \pm 0.02$ [19], $z=1.97 \pm 0.08$ [20], $z=2.04 \pm 0.03$ [21], $z=2.04 \pm 0.01$ [22], $z=2.032 \pm 0.004$ [23]. Данные значения индекса $z$ находятся в хорошем согласии с полученными в настоящей работе результатами и убеждают, что предсказываемое нами значение $z=2.0237 \pm 0.0055$ является достаточно надежным.

Что касается двумерной однородной модели Изинга, то численные исследования ее критической динамики в отличие от аналогичных исследований трехмерной модели характеризуются гораздо бо́льшим разбросом получаемых значений для динамического критического индекса: $z=2.14 \pm 0.02$ [24], $z=2.13 \pm 0.03$ [25], $z=2.076 \pm 0.005$ [26], $z=2.24 \pm 0.04$ [27], $z=2.24 \pm 0.07$ [28], $z=2.16 \pm 0.04$ [29], $z=2.1667 \pm 0.0005$ [30]. Такой разброс значений $z$ во многом объясняется тем, что критическое поведение двумерной модели Изинга характеризуется как гораздо бо́льшими по сравнению с трехмерной моделью амплитудами флуктуаций параметра порядка в критической области, так и более значительными эффектами критического замедления.

Применение метода высокотемпературного разложения к описанию критической динамики двумерной модели Изинга дает значение $z=2.125$ [31]. 
Таким образом, полученные в цитируемых работах для двумерной модели Изинга значения индекса $z$ лежат в достаточно широком интервале $2,07 \leqslant z \leqslant 2,31$, в то время как полученные в настоящей работе значения находятся на его нижней границе. Это поднимает ценность полученных нами значений как ориентиров для будущих исследований.

Проведем теперь сопоставление рассчитанных в данной работе значений динамического критического индекса для неупорядоченных систем с результатами компьютерного моделирования критической динамики неупорядоченной трехмерной модели Изинга: $z=2.19 \pm 0.07$ для систем со спиновой концентрацией $p=0.95$, $z=2.20 \pm 0.08$ при $p=0.8, \quad z=2.58 \pm 0.09$ при $p=0.6$ и $z=2.65 \pm 0.12$ при $p=0.4[20] ; z=2.16 \pm 0.01$ при $p=0.95, z=2.232 \pm 0.004$ при $p=0.9, z=2.38 \pm 0.01$ при $p=0.8$ и $z=2.93 \pm 0.03$ при $p=0.6$ [32]. Данные результаты моделирования критической динамики находятся в достаточно хорошем согласии с результатами теоретико-полевого расчета с использованием методов суммирования лишь для слабо неупорядоченных систем с $p \geqslant 0.8$, в то время как для сильно неупорядоченных систем наблюдается заметное расхождение результатов. При этом следует отметить, что результаты РГ-описания критического поведения неупорядоченных систем справедливы лишь в области слабой неупорядоченности. Для объяснения наблюдаемой при компьютерном моделировании зависимости индекса $z$ от величины структурного беспорядка в работе [20] была предложена гипотеза ступенчатой универсальности, согласно которой в системах при спиновых концентрациях выше порога спиновой перколяции может наблюдаться несколько типов различного критического поведения в зависимости от того, существует ли в системе лишь один спиновый протекающий кластер, как в случае слабо неупорядоченных систем, или наряду со спиновым протекающим кластером реализуется и примесный протекающий кластер, как в случае сильно неупорядоченных систем с существованием переходных режимов между областями. В работе [32] автор, исходя из концепции универсальности критического поведения неупорядоченных систем и независимости асимптотического значения индекса $z$ при $L \rightarrow \infty$ от степени беспорядка, на основе приведенных выше эффективных значений индекса получил его асимптотическое значение $z=2.4 \pm 0.1$. Однако данное значение индекса находится в сильном несоответствии с полученными в настоящей работе результатами. В работе [33] был проведен анализ численного исследования критической динамики трехмерной модели Изинга со спиновой концентрацией, изменяющейся в широком интервале. Авторы, предполагая универсальность критического поведения неупорядоченных систем, выделили асимптотическое значение индекса $z=2.62 \pm 0.07$ с учетом эффектов влияния ведущих поправок к скейлинговой зависимости для динамической восприимчивости системы. При этом полученное в работе [33] значение индекса поправки к скейлингу $\omega=0.50 \pm 0.13$ находится в сильном несоответствии с результатами теоретико-полевого расчета статических критических индексов, осуществленного с применением методов суммирования в работе [9] и давшего значение $\omega=0.25 \pm 0.10$, а также с результатами численного исследования статического критического поведения той же модели [34], проведенного также с учетом эффектов влияния ведущих поправок к скейлинговой зависимости термодинамических величин и корреляционных функций с $\omega=0.37 \pm 0.06$. При 
осуществленных в работе [33] аппроксимациях наибольшими погрешностями характеризовались результаты для слабо неупорядоченных систем. Заметим также, что полученное в работе [33] значение динамического критического индекса $z$ находится в еще более сильном несоответствии с результатами настоящей работы.

Экспериментальное исследование критической динамики неупорядоченного изинговского антиферромагнетика $\mathrm{Fe}_{0.46} \mathrm{Zn}_{0.54} \mathrm{~F}_{2}$ было осуществлено в работе [18], в которой было получено значение $z=1.7 \pm 0.2$. Данный результат находится в противоречии как с результатами теоретико-полевого расчета с использованием методов суммирования, так и с приведенными выше результатами компьютерного моделирования. Это несоответствие, возможно, обусловлено высокой концентрацией примеси в исследованном образце и наличием крупномасштабных неоднородностей в нем, что существенно сказывается на характеристиках неравновесного критического поведения. Однако в другой экспериментальной работе [35] по исследованию критической динамики слабо разбавленного изинговского магнетика $\mathrm{Fe}_{0.9} \mathrm{Zn}_{0.1} \mathrm{~F}_{2}$ в результате проведенного методом мессбауэровской спектроскопии высокопрецизионного измерения динамического уширения мессбауэровских линий было получено значение динамического критического индекса $z=2.18 \pm 0.10$. Данное значение находится в прекрасном соответствии с результатами проведенных нами расчетов. Тем не менее возникает настоятельная необходимость дополнительных как экспериментальных, так и численных исследований критической динамики неупорядоченных изингоподобных систем. Полученные в данной статье результаты расчета динамического критического индекса для неупорядоченных систем будут служить ориентирами для подобных исследований.

Благодарности. Исследования поддержаны РФФИ (гранты № 04-02-17524, 0402-39000 и 05-02-16188).

\section{Список литературы}

[1] P. C. Hohenberg, B. I. Halperin, Rev. Mod. Phys., 49 (1977), 435.

[2] R. Bausch, H.K. Janssen, H. Wagner, Z. Phys. B, 24 (1976), 113; C. De Dominicis, E. Brézin, J. Zinn-Justin, Phys. Rev. B, 12 (1975), 4945; H. В. Антонов, А.Н. Васильев, TMФ, 60 (1984), 59; B.I. Halperin, P. C. Hohenberg, S. Ma, Phys. Rev. Lett., 29 (1972), 1548; B. I. Halperin, P. C. Hohenberg, E. D. Siggia, S. Ma, Phys. Rev. B, 13 (1976), 2110.

[3] В. В. Прудников, А.Н. Вакилов, ЖКЭТФ, 101 (1992), 1853.

[4] В.В. Прудников, А. В. Иванов, А. А. Федоренко, Писъма в ЖЭТФ, 66 (1997), 793.

[5] В. В. Прудников, С. В. Белим, А. В. Иванов и др, ЖКЭТФ, 114 (1998), 972.

[6] G. A. Baker, B. G. Nickel, M. S. Green, D. I. Meiron, Phys. Rev. Lett., 36 (1976), 1351; Phys. Rev. B, 17 (1978), 1365 .

[7] J. C. Le Guillou, J. Zinn-Justin, Phys. Rev. Lett., 39 (1977), 95; Phys. Rev. B, 21 (1980), 3976

[8] S. A. Antonenko, A. I. Sokolov, Phys. Rev. B, 51 (1995), 1894.

[9] A. Pelissetto, E. Vicari, Phys. Rev. B, 62 (2000), 6393.

[10] J. Zinn-Justin, Quantum field theory and critical phenomena, Clarendon Press, Oxford, 1996; А.Н. Васильев, Квантовополевая ренормгруппа в теории критического поведения и стохастической динамике, ПИЯФ, С.-Петербург, 1998.

[11] В. С. Доценко, УФН, 165 (1995), 481. 
[12] Л.Н. Липатов, ЖЖЭТФ, 72 (1977), 411.

[13] E. Brézin, J. C. Le Guillou, J. Zinn-Justin, Phys. Rev. D, 15 (1977), 1544.

[14] Г. Харди, Расходящиеся ряды, Иностр. лит., М., 1951; Д. И. Казаков, О.В. Тарасов, Д. В. Ширков, ТМФ, 38 (1979), 15; Д.И. Казаков, В. С. Попов, ЖЖЭТФ, 122 (2002), 675; И. М. Суслов, ЖЭТФ, 120 (2001), 5.

[15] J. Honkonen, M. V. Komarova, M. Yu. Nalimov, Nucl. Phys. B, 714 (2005), 292; 707, 493; hep-th/0406168.

[16] C. M. Bender, T. T. Wu, Phys. Rev., 184 (1969), 1231.

[17] J. Čižek, E. R. Vrscay, Int. J. Quant. Chem., 21 (1982), 27.

[18] D. P. Belanger, B. Fagaro, V. Jaccarino et al, J. Phys. Collque C8, 49 (1988), 1229.

[19] R. B. Pearson, J. L. Richardson, D. Toussaint, Phys. Rev. B, 31 (1985), 4472.

[20] В. В. Прудников, А.Н. Вакилов, ЖЖЭТФ, 103 (1993), 962.

[21] S. Wansleben, D. P. Landau, Phys. Rev. B, 43 (1991), 6006.

[22] U. Gropengiessen, Physica A, 213 (1995), 308.

[23] P. Grassberger, Physica A, 214 (1995), 547.

[24] C. Kalle, J. Phys. A, 17 (1984), L801.

[25] J. K. Williams, J. Phys. A, 18 (1985), 49.

[26] M. Mori, Y. Tsuda, Phys. Rev. B, 37 (1988), 5444.

[27] P. H. Poole, N. Jan, J. Phys. A, 23 (1990), L453.

[28] V. V. Prudnikov, O. N. Markov, Europhys. Lett., 29 (1995), 245.

[29] F. Wang, N. Hatane, M. Suzuki, J. Phys. A, 28 (1995), 4543.

[30] M. P. Nightingale, H. W. J. Blöte, Phys. Rev. B, 62 (2000), 1089.

[31] Z. Racz, M. F. Collins, Phys. Rev. B, 13 (1976), 3074.

[32] H.-O. Heuer, J. Phys. A, 26 (1993), L341.

[33] G. Parisi, F. Ricci-Tersenghi, J. J. Ruiz-Lorenzo, Phys. Rev. E, 60 (1999), 5198.

[34] H. G. Ballesteros, L. A. Fernandez, V. Martin-Mayor et al, Phys. Rev. B, 58 (1998), 2740 .

[35] N. Rosov, C. Hohenemser, M. Eibschutz, Phys. Rev. B, 46 (1992), 3452.

Поступила в редакцию 25.VII.2005 г., после доработки 8.IX.2005 г. 